\title{
Exploring Family Multi-type Maltreatment, Social Support, and Externalizing and Internalizing Problems Among Asian and Asian American College Students
}

\author{
Yoko Baba ${ }^{1}$, James D. Lee ${ }^{2} \&$ Michael E. Vallerga ${ }^{1}$ \\ ${ }^{1}$ Justice Studies Department, San José State University, San José, CA, U.S.A. \\ ${ }^{2}$ Faculty Affairs, San José State University, San José, CA, U.S.A. \\ Correspondence: Yoko Baba, Department of Justice Studies, San José State University, San José, CA, \\ 95192-0050, U.S.A. E-mail: yoko.baba@ sjsu.edu
}

Received: July 31, 2020

Accepted: August 26, 2020

Online Published: October 1, 2020

doi:10.20849/ajsss.v5i3.780

URL: https://doi.org/10.20849/ajsss.v5i3.780

\begin{abstract}
Exposure to family violence as a child has a detrimental long-term impact on one's life. This relationship is under-researched in Asian populations in the United States or in Asian countries. This study examined long-term effects of maltreatment, including interparental violence and child maltreatment on externalizing and internalizing problems experienced by Asian and Asian American college students. We also explored protective effects of social support against the negative consequences of family maltreatment. Surveying 542 college students in Hong Kong, South Korea, Japan, and the United States, we measured effects of family maltreatment on problem outcomes and examined the role of social support. Exposure to dual harm of family maltreatment (i.e., intraparental violence and child maltreatment) increased students' externalizing problems compared to exposure to one type of family maltreatment, but no differences in internalizing behaviors were found. Effects of social support from parents and peers on externalizing and internalizing problems were neither moderating nor mediating, but direct. Those who received parental support had fewer externalizing behaviors, but effects of peer support were not significant. In contrast, those who obtained parental and peer support showed lower levels of internalizing mental health concerns. Surprisingly, men exhibited more mental health issues than women. Exposure to dual harm increases behavioral problems, but family support can help repair damage among Asian and Asian American college students. The relationship between abuse and problem outcomes was similar across countries, indicating common psychological processes.
\end{abstract}

Keywords: multi-type maltreatment, externalizing behavior, internalizing behavior, social support, Asian college students, Asian American college students

\section{Introduction}

Family multi-type maltreatment - experiences of multiple forms of child abuse and neglect (Higgins \& McCabe, 2001) - including interparental violence and child maltreatment, exists in all countries, representing a major human rights and public health problem (Bouhours \& Broadhoust, 2015; Heise \& Garcia-Moreno, 2002). Family violence is the leading cause of injury among women and children internationally, including in Asian countries (Tsokodavi, 2010; UNICEF, 2014). Research conducted in the United States showed that exposure to family violence as a child had a significant detrimental long-term impact on one's life (Gewirtz \& Edleson, 2004). Children exposed to both intimate partner violence and child maltreatment exhibited more difficulties in areas of social, emotional, behavioral, and cognitive functioning than non-exposed peers (Gewirtz \& Edleson, 2004; Wolfe, Crooks, Lee, McIntyre-Smith, \& Jaffe, 2003).

Those from abusive families experience physical health problems (e.g., injuries, heart problems, and migraine headaches), mental health problems (e.g., post-traumatic stress disorder [PTSD], depression, and anxiety), and even premature death (U.S. Department of Health and Human Services [USDHHS], 2018). Community well-being is also reduced through perpetration of further violence, trauma, fear of crime, and increased spending on criminal justice and social welfare (Gilbert et al, 2009). Many survivors of family violence and maltreatment either quit or are forced to leave their jobs, resulting in lifetime income losses of nearly $\$ 250,000$ 
person (USDHHS, 2018). Some abused women and children are also forced to leave their homes and become homeless (USDHHS, 2018; Ferguson, 2009).

Early studies of mental health problems (i.e., internalizing problems) of children who have been exposed to family violence and maltreatment were mostly carried out with Western populations (Haj-Yahia \& de Zoysa, 2008). Recently, a limited number of studies have been conducted in non-Western societies among Asians, showing similar patterns to those in the United States.

In Hong Kong, intimate partner violence is strongly associated with child physical maltreatment, and this dual family harm leads to higher rates of feeling threatened, lower self-esteem (internalizing), and aggression and violence (externalizing; Chan, 2011). In Taiwan, those experiencing multi-type family maltreatment demonstrated more internalizing and externalizing problems than those experiencing only one type of maltreatment (Shen, 2009). However, among South Korean young adults, exposure to a single type of maltreatment resulted in equally high levels of internalizing and externalizing problems as those who experienced dual family harm (Han, Choi, \& Jung 2016).

Family maltreatment strains the fundamental human need to belong (Baumeister \& Leary, 1995), but social support eases distress and promotes prosocial engagement (Lee, Chung, Back, \& Batzolboo, 2014). Cultural differences also complicate understanding the effects of these dynamics across Eastern and Western cultures. For example, fidelity to family for support may allow greater social competitiveness among Asian students, and seeking belonging among friends may hinder individuation among Western students. Characterized by collectivism and interdependence, Asian societies may encourage the sharing of stressful problems. However, adherence to group or family harmony may discourage the use of a social support network in coping with stress because personal beliefs and needs are secondary to social norms and relationships (Taylor et al., 2004; Shen 2009).

Kim, Sherman, and Taylor (2008) observed that even though Asian and Asian Americans are distinctive cultural groups, they share some cultural heritage; the shared cultural experiences underlie a tendency to not seek social support. In contrast, in Western cultures, such as the United States, individuals are viewed as independent and encouraged to act according to their own interests. Thus, Westerners are more likely to seek assistance from their social networks to deal with family problems.

Social support has been found to mitigate effects of stress on psychological and behavioral problems, but evidence of that relationship is inconclusive, and more research is needed on that topic (Bokhorst, Summer, \& Westenberg, 2010; Kennedy, Bybee, Sullivan, \& Greeson, 2010; Sangalang \& Gee, 2012). Social support may disrupt the cycle of violence. Ironically, parents who abused their children, via social support, may play a role in reducing the likelihood of subsequent violence by their abused children.

To address the need for more research among Asian and Asian American college students on this topic, and more through a comparative lens, we examine the long-term impacts of family multi-type maltreatment (i.e., interparental violence and child maltreatment) on externalizing and internalizing problems of Asian college students in Hong Kong, South Korea, and Japan and Asian American college students. Furthermore, our investigation also includes the role of social support in the effects of externalizing and internalizing problems. This approach may advance our understanding of cultural variations in outcomes stemming from family multi-type maltreatment.

\section{Literature Review}

\subsection{Family Violence and Maltreatment}

Children in violent homes are at a higher risk for being abused than children in non-violent homes. More than $60 \%$ of children are exposed to direct or indirect abuse each year in the United States (Finkelhor, Turner, Ormrod, Hamby, \& Kracke 2009). Children indirectly exposed to intimate partner violence are often direct victims of other kinds of maltreatment in their homes, and this dual harm is experienced in as many as $40 \%$ of families (Appel \& Holden, 1998; Straus \& Smith, 1990). This percentage closely corresponds with those of Hong Kong (Chan, 2011).

Children who were exposed to multi-type maltreatment are more likely to be disobedient at home and school and more likely to show less social competence, including lower relationship quality and school performance when compared to non-exposed children (Stiles, 2002). Additional outcomes include aggressive and antisocial behaviors (i.e., externalizing behaviors), fearful and inhibited behaviors (i.e., internalizing behaviors), drinking problems (Hamburger, Leeb, \& Swahn, 2008; Luster, Small, \& Lower, 2002), depression and delinquency (Moylan et al., 2010), and problems at school (Kiesel, Piescher, \& Edelson, 2016). 
Research comparing outcomes of children who have witnessed interparental violence and those who experienced direct child abuse is unsettled. Effects on antisocial behavior in childhood appear to be greater for those directly abused than for those who witness interparental violence (Wilson, Stover, \& Berkowitz, 2009). Abused children also experience severe negative emotions, which account for their increased risk of internalizing behavior problems (Salzinger et al., 2002). However, exposure to both types of family maltreatment - or dual harm - in childhood might have a greater adverse effect on children than either single type alone (Eriksson \& Mazerolle, 2015). Additionally, children exposed to dual harm have a much higher risk of negative psychosocial (i.e., internalizing) and behavioral (i.e., externalizing) problems compared to those not exposed to maltreatment (Herrenkohl, Sousa, Tajima, Herrenkohl, \& Mylan, 2008). Dual harm not only has an immediate negative effect on children, but also a long-term detrimental impact on adult adjustment (Gewirtz \& Edleson, 2004). Among Taiwanese college students, dual harm had a more lasting adverse impact on self-esteem than the impact of one type of maltreatment or none at all (Shen, 2009).

Cho (2012) notes the few comparative studies on multi-type maltreatment experienced by Asian and Asian American adolescents. Most were conducted to study intimate partner violence among Chinese Americans (Hicks, 2006; Yick, 2000), South Korean Americans (Kim \& Sung, 2000; Lee, 2007), and Japanese immigrants (Yoshihama, 1999). As such, long-term effects of family maltreatment and dual harm among Asian and Asian American adolescents needs more attention (Chen et al., 2012; Shen, 2009).

\subsection{Intergenerational Transmission of Violence}

The intergenerational transmission of violence perspective (Isaac, 2015), is useful for explaining the relationship between children's exposure to familial violence and maltreatment (whether indirect, direct, or both), and behavioral outcomes. With roots in social learning theory (Akers, 1998), the perspective asserts that violence is transmitted through vicarious observations and direct experiences (Eriksson \& Mazerolle, 2015), resulting in rewards and punishments for violent thoughts and behaviors. Survivors' cognitive and behavioral norms are infused with violent schemas that may play out in other contexts well into adulthood (Gover, Jennings, Tomsich, Park, \& Rennison, 2011; Isaac, 2015). Reinforcement of witnessing or experiencing violence in the family varies with situational family dynamics and social roles, making specific outcomes difficult to predict (Eriksson \& Mazerolle, 2015; Wilson, et al., 2009). However, unless disrupted, violent social patterns and behaviors learned in childhood remain central in survivors' lives.

Children who witness intimate partner violence sometimes model parental behavior in interactions with other people, increasing risk of externalizing behavior problems (Salzinger et al., 2002), including violent perpetration in dating relationships (Carr \& Vandeusen, 2002). Furthermore, child maltreatment (being an abuse survivor) profoundly increases antisocial behavior in adolescence (Wilson, et al., 2009). Finally, child survivors with severe negative emotions experience anger in interactions with other people and have higher risk for internalizing behavior problems (Salzinger, et al., 2002).

\subsection{Role of Social Support}

Social support is information from others that one is cared for, loved, esteemed, and a member of a network of mutual obligations (Cobb, 1976). Research in the United States found that social support helps individuals reduce and cope with physical and mental stress (Thoits, 2011; Talwar \& Mohd Fadzil, 2013). The absence of social support leads to emotional, academic, and financial stress (Talwar \& Mohd Fadzil, 2013). In the United States, family often serves as a model for coping behavior and provides a sense of safety, while friends reduce isolation and assist coping efforts (Vernberg, Greca, Silverman, \& Prinstein, 1996). However, it is unclear whether social support functions the same way in non-Western societies (Matsuda, Tsuda, Kim, \& Deng, 2014). Shen (2009) found that parental and peer social support mediated between childhood dual harm and low self-esteem among Taiwanese college students. There is a "double whammy" effect of maltreatment and poor relationship quality (p. 787).

\subsection{Cultural Values and Social Support}

The salience and value of sources of social support vary according to both cultural values and age. Asian cultural values such as collectivism emphasize the maintenance of group relations, stress conformity and the well-being of others, and suppress deviance and mental concerns (Anderson \& Mayes, 2010; Keen, Pickering, Wieczorek, \& Blaszczynski, 2015; Sangalang \& Gee, 2012). As such, seeking out social support is discouraged because personal beliefs and needs are secondary to social norms and relationships (Sangalang \& Gee, 2012). Another value, "saving face" also discourages maltreated family members from seeking help from friends and family (Kopp, 2010, para. 2) and urges the family unit to remain intact (Meyers, 2006; Keen et al., 2015). Therefore, families and friends may be viewed as the source of strain rather than support in Asian cultures. 
Yoshihama and Dabby (2009) showed that Japanese immigrant women born in Japan, compared with Japanese American women, were less likely to seek help from friends and confront their partners. This suggests that those who are born in Japan were more constrained by the cultural expectations and traditions of their ethnic background than those born in the U.S., thereby making them more vulnerable and intensifying negative impacts on their lives (Yoshihama, 2002). Lee and Hadeed (2009) demonstrated that in South Korean immigrant communities, positive support from families, relatives, and friends was more likely to reduce life stress, distress symptoms, and spousal violence. Han et al. (2016) found that, for South Korean college students who experienced family violence, social support from parents, close friends, teachers, and classmates reduced internalizing mental health outcomes, helping "them rebuild their sense of self" (p. 831). However, they did not find the effect of social support on externalizing behavior. This was consistent with research from the United States showing a lack of impact of social support from peers and teachers on externalizing behavior which was instead more related to poor relationships with parents (White \& Ren, 2012).

\subsection{Age and Social Support}

The influence of social support on outcomes of family maltreatment may also vary with age. At a younger age, parental support takes a primary role, while in the case of college students, peer support comes to the fore (Tremblay, Hébert, \& Piché, 1999). Children are more likely than adolescents to get support from adults and were more satisfied with support received from adults. Ezzell, Swenson, Cupit, and Brondino (2000) reported that among physically abused children in the United States, both parental and peer support reduced internalizing problems, but not externalization. Parents assume a nurturing role, providing children with a sense of security and protection, while peer relationships decreased feelings of loneliness, increased sense of belonging, and validated self-worth (Parker \& Asher, 1993). Although family and peer support is important to children undergoing stress, more support is needed to prevent aggression.

In contrast, adolescents are less likely to feel supported by parents, but more likely to view friends as support resources (Feiring, Taska, \& Lewis, 1998). They spend less time with their parents and rely less on them for companionship and emotional support. Support from parents did not always facilitate healthier functioning following abuse discovery. Instead, adolescents spend more unsupervised time with peers, which may not protect against psychological distress, but rather it can be a risk factor. Victimized youths who perceived friends as their major source of support were particularly at risk for poor adjustment, including internalizing behaviors such as shame and self-blame (Feiring et al., 1998). It is because when they have suffered stress, the comfort of peer support is not enough to avoid internalizing and externalizing problems (Bal et al., 2003). Feiring et al. (1998) noted if the supportive peer group engaged in aggressive behavior, then the youth, although feeling supported, might also be more likely to engage in aggression. Thus, social support may not mitigate effects of stressful events and coping among adolescents.

However, adolescents who do feel supported by their parents have less psychological distress. They show reduced symptoms of depression, anxiety, anger, and hostility, regardless of the severity of child abuse experienced (Folger \& Wright, 2013; Holahan, Valentiner, \& Moos, 1995).

\subsection{Ways Social Support May Affect Behavior}

Although past research confirmed that social support plays an important role in the reduction of psychological and behavioral problems, it is inconclusive about whether social support serves as a mediator or moderator (Bokhorst et al., 2010; Kennedy, Bybee, Sullivan, \& Greeson, 2010; Sangalang \& Gee, 2012). Few studies examine whether social support mediates or moderates the relationship between child abuse and adult mental and physical health (Herrenkohl et al., 2016).

\subsubsection{Mediating Effect of Social Support}

Social support may mediate the relationship between family violence, maltreatment, and positive coping (Herrenkohl et al., 2016; James \& Mennen, 2018; Owen et al., 2008). Herrenkohl et al. found that social support mitigates the relationship between child abuse and adult mental and physical outcomes. Owen et al.'s examination of 8-12 year-old African American children showed that both family and peer support mediates the effect of intimate partner conflict on their social functioning. James and Mennen also found that for a sample of mostly Latino and African American youth and young adults between 14 and 22 years old, social support (measured as positive interaction with friends) mediates the relationship between maltreatment and internalizing problems. Social support from peers has a negative association with emotional problems among youth and young adults who experienced child abuse. 


\subsubsection{Moderating (Buffering) Effect of Social Support}

Findings from research exploring social support as a moderating or protective factor have been mixed (Folger \& Wright, 2013). Some studies found that social support buffers against negative outcomes abuse survivors (Schumm, Briggs-Phillips, \& Hobfoll, 2006). Davidson and Demaray (2007) showed that social support played a buffering role between middle school students' victimization and internalization problems from bullying. Social support from teachers and classmates for boys, and parental support for girls moderated the relationship between bullying victimization and internalizing problems.

However, other studies that had the ability to do so found no moderating effects (Herrenkohl et al., 2016). Bal et al. (2003) also showed that, for adolescents, social support did not moderate between a stressful event and coping. Likewise, Folger and Wrights (2013) did not find moderating effects when abuse experiences were more severe and repeated. Internalizing problems persisted despite social support from families, partly because support from families exhibiting high levels of aggression might not be beneficial to children and because they were more likely to suffer from high levels of stress, which outweighed positive support (McCloskey, Figueredo, and Koss, 1995).

Few studies examine social support as a mediating or moderating influence for Asian and Asian American adolescents. A rare example, Yoshihama and Horrocks' (2002) study of women abuse survivors of Japanese descent, found no interaction effects between abuse and social support on PTSD symptoms.

\section{Hypotheses and Research Questions}

This research aims to understand the effects of family maltreatment on Asian and Asian American college students' externalizing and internalizing behaviors, as well determine the role of social support (directly, mediating, or moderating) in that process. We also explore if those who receive higher levels of parental and peer support experience lower levels of externalizing and internalizing problems. Furthermore, we examine if cross-cultural differences in externalizing and internalizing problems exist. We formulated the following 2 hypotheses and 3 research questions:

3.1 Hypothesis 1. College students who were exposed to some kind of family maltreatment (interparental violence, child maltreatment, or dual harm) will have higher levels of a) externalizing problems and b) internalizing mental health concerns than those not exposed to maltreatment, controlling for social support, country of origin, and gender.

3.2 Hypothesis 2. College students who had dual harm will have higher levels of externalizing and internalizing problems than those who experienced any single form of family maltreatment, controlling for social support, country of origin, and gender.

3.3 Research Question 1. Does social support play a direct, mediating, or moderating role in the relationship between family maltreatment and externalizing and internalizing problems?

3.4 Research Question 2. Do Asian and Asian American college students who receive higher levels of parental and peer support experience lower levels of externalizing and internalizing problems, controlling for family maltreatment, country of origin, and gender?

3.5 Research Question 3. Do cross-cultural differences in externalizing and internalizing problems exist, controlling for family maltreatment, social support, and gender?

\section{Methods}

After receiving approval from the university's institutional review board, we conducted the present study using a survey questionnaire. To have culturally sensitive, reliable, and valid measurements, we modified all survey instruments by incorporating the suggestions made by colleagues ${ }^{1}$ in the respective countries. We also employed a pilot study in each country, which had internally consistent results. Because of known cultural differences among Asian countries, we did not intend to generalize our findings to college students in all Asian countries and Asian American college students.

\subsection{Participants}

The sample consists of 542 college students, comprising 155 (28.6\%) at a Hong Kong university, $96(17.7 \%)$ at one of three South Korean universities, $172(31.7 \%)$ at one of three Japanese universities, and $119(22.0 \%)$ at a U.S. university. In total, the sample was drawn from eight universities across four countries. Of the total participants, $156(28.8 \%)$ were men and $386(71.2 \%)$ women. 


\subsubsection{Hong Kong, South Korean, and Japanese Students}

In Hong Kong, South Korea, and Japan, each professor distributed hard copies of the survey questionnaires to their students during class time and asked them to return it after the class or during the next class.

\subsubsection{Asian American Students}

Asian American students in general psychology classes were recruited at a large Western university, using the SONA online system, which allowed researchers to manage their research in a cloud-based environment (http://www/soma-systems.com/about.aspx). To participate in the study, Asian American students had to be (a) 18 or older, (b) undergraduate students at the university, (c) American-born or foreign-born (born Chinese, Korean, Japanese or Vietnamese). If foreign-born, participants had to have arrived in the United States before the age of 12 , which suggested they were assimilated into the local and national American culture and society.

\subsection{Survey Instrument and Data Collection}

All instruments were originally written in English and then professionally translated between October and December 2012. Data were collected early March to mid-March 2013.

\subsection{Measures}

Variables included externalizing and internalizing problems, family maltreatment, social support, country of origin, and gender.

\subsubsection{Dependent Variables}

\subsubsection{Externalizing and Internalizing Problems}

To measure externalizing and internalizing problems, Achenbach's (1991) Child Behavior Check List was used. Externalizing problems included twenty-two items, such as "argue a lot," "get along badly with my family," "physically attack people," and "threaten to hurt people." Internalizing problems included twenty-one items, such as "lonely," "worthless or inferior," "nervous or tense," and "unhappy, sad, or depressed." For each question, participants were asked to rate the frequency with which they experienced each behavior problem over the past six months using the following answer format: $0=$ not true, $1=$ somewhat or sometimes true, and $2=$ very true or often true. A higher number indicates more externalizing and internalizing problems. Among the four countries combined, Cronbach's alpha was .88 for externalizing problems and .91 for internalizing problems.

\subsubsection{Independent Variables}

\subsubsection{Family Maltreatment}

Family maltreatment consists of a) interparental violence and b) child maltreatment. The questions used the problematic parental terms of "mother" and "father," but instructed participants to include step-parents when recounting these experiences.

a) Interparental violence. Interparental violence was measured by the Conflict Tactics Scale for parental violence (Straus, Hamby, Boney-McCoy, \& Sugarman, 1996). Participants answered how often they were exposed to various violent acts between their parents, such as mental or physical abuse, before they turned 18 years old.

Witness of father abusing mother and witness of mother abusing father. These two variables each consisted of two types of abuse (i.e., mental and physical abuse), which comprised a single scale, 1) witness father, and 2) witness mother for each country.

Witness of father (or mother) abusing mother (or father) mentally and physically. Six questions from the mental abuse scale for father (or mother) and eight questions from the physical abuse scale for father (or mother) were included. Participants were asked to indicate how many times their father (or mother) committed mental and physical abuse upon their mother (or father) when participants were in elementary through high school. The response options were 1 (almost daily) to 7 (never). Response categories were reverse coded, so higher numbers indicate higher frequencies. Among the four countries combined, Cronbach's alpha was: for mental abuse, .89 for father and .85 for mother; and for physical abuse, .97 for father and .95 for mother.

The correlation between witnessing father abusing mother and mother abusing father (mental and physical) (combined four countries) was strong and positive, $\mathrm{r}(490)=.76, \mathrm{p}<.001$. For each country, the correlation between witnessing father abusing mother and mother abusing father was strong (except in the United States, which was moderate) and positive ( $\mathrm{p}<.001)$ : $\mathrm{r}(140)=.81$ for Hong Kong, $\mathrm{r}(82)=.88$ for South Korea, $\mathrm{r}(155)=.84$ for Japan, and $\mathrm{r}(107)=.54$ for the United States. 
b) Child maltreatment. Child maltreatment was measured by the Parent-Child version of Contact Tactics Scales for child maltreatment history (Straus, Hamby, Boney-McCoy, \& Sugarman, 1998). Respondents reported the frequency of mental and physical maltreatment by their father and mother before they turned 18 years old.

Child maltreatment by father and child maltreatment by mother. These two variables each consisted of two types of maltreatment (i.e., mental and physical maltreatment), which comprised a single scale, 1) child maltreatment by father, and 2) child maltreatment by mother for each country.

Mental and physical maltreatment by father (or mother). Five questions from the mental maltreatment scale and six questions from the physical maltreatment scale were included. Participants were asked to indicate how frequently they were mentally and physically maltreated by the parent when they were in elementary through high school. The answer category was 1 (almost daily) to 7 (never). Response categories were reverse coded, so higher numbers indicate higher frequencies. Among the four countries combined, Cronbach's alpha was: for mental maltreatment, .88 for father and .82 for mother; and for physical maltreatment, .87 for father and .86 for mother.

The correlation between child maltreatment (mental and physical) by the father and by the mother (combined four countries) was moderate and positive, $\mathrm{r}(473)=.55, \mathrm{p}<.001$, indicating common co-occurrence of parents being abusive. For each country, the correlation between child maltreatment by the father and by the mother was positive, but ranged from weak (the United States), to moderate (Hong Kong and Japan), to strong (South Korea): $\mathrm{r}(134)=.59$ for Hong Kong, $\mathrm{r}(82)=.75$ for South Korea, $\mathrm{r}(149)=.69$ for Japan, and $\mathrm{r}(102)=.26$ for the United States.

Four groups of family maltreatment exposure. To test whether dual exposure to interparental violence and child maltreatment has a greater association with externalizing and internalizing problems than single exposure to either interparental violence or child maltreatment, the sample was divided into four mutually exclusive groups (see Moylan et al., 2010): 1) no violence exposure group ( $\mathrm{n}=11,2.0 \%$ of sample); 2) witness of interparental violence only group which includes domestic violence by father only, mother only, and both father and mother $(\mathrm{n}=25,4.6 \%) ; 3)$ child maltreatment only group, which includes child abuse by father only, mother only, and both father and mother $(\mathrm{n}=193,34.1 \%)$; and 4) dual harm group, which includes interparental violence and child maltreatment by father only, mother only, and both father and mother $(n=320,58.3 \%)$. Moylan et al. (2010) found that $68 \%$ of their sample (adolescents) experienced some type of maltreatment, while $88.8 \%$ of our Asian and Asian American respondents (college students) experienced either exposure to interparental violence, child maltreatment, or dual harm. We created three dummy variables, which are shown in statistical analyses later in the paper: interparental violence; child maltreatment; and dual harm. No maltreatment is the omitted category.

\subsubsection{Social Support}

The primary instrument for social support was the Child and Adolescent Social Support Scale [CASSS], which is a multi-dimensional scale measuring perceived social support from four sources: parents, teachers, classmates, and friends (Malecki \& Demaray, 2002). Past research indicated that classmate support was stable over time, but teacher support decreases with age (Bokhorst et al., 2010). Our sample consisted of college students, so we included parental and peer support only.

Social support from parents and close friends. The questions asked participants' current experience with their parents in seven items and their close friends in eight items using a 6-point scale: $1=$ never, $2=$ very rarely, $3=$ rarely, $4=$ occasionally, $5=$ very frequently, and $6=$ always. Among the four countries combined, Cronbach's alpha was: .90 for social support from parents and .96 for social support from close friends.

\subsubsection{Additional Variables}

\subsubsection{Gender}

This question asked whether participants were men (coded as 1$)$ or women (coded as 0$)$.

\subsubsection{Country of Origin}

This indicated the country in which participants attended university. Response categories were 1 for Hong Kong, 2 for South Korea, 3 for Japan, and 4 for the United States. We created three dummy variables: Hong Kong; South Korea; and Japan.

\section{Results}

Hierarchical regression analysis was used to show if variables explain a statistically significant amount of variance in the dependent variable (Kim, 2018). This analysis allows us to build several regression models by 
adding variables to a previous model at each step and to determine whether newly added variables show an improvement.

Table 1 presents family maltreatment by gender. Women experienced more dual harm and child maltreatment than men. Contrastingly, men witnessed more interparental violence than women and more men than women were not exposed to any maltreatment.

Table 1. Number and percentage of cases for family maltreatment by gender

\begin{tabular}{lccccc}
\hline & $\begin{array}{c}\text { No } \\
\text { maltreatment }\end{array}$ & $\begin{array}{c}\text { Interparental } \\
\text { violence }^{\mathrm{a}}\end{array}$ & $\begin{array}{c}\text { Child }^{\text {maltreatment }} \\
\text { ben }^{\mathrm{b}}\end{array}$ & Dual Harm & Total \\
\hline Men & $7(64 \%)$ & $19(76 \%)$ & $50(27 \%)$ & $93(29 \%)$ & $169(31 \%)$ \\
Tomen & $4(36 \%)$ & $6(24 \%)$ & $138(73 \%)$ & $225(71 \%)$ & $373(69 \%)$ \\
Total & $11(2 \%)$ & $25(5 \%)$ & $188(35 \%)$ & $318(55 \%)$ & $542(100 \%)$ \\
\hline
\end{tabular}

${ }^{a}$ Interparental violence includes father only, mother only, and father and mother

${ }^{\mathrm{b}}$ Child maltreatment includes father only, mother only, and father and mother

${ }^{\mathrm{c}}$ Dual harm consists of interparental violence and child maltreatment

Table 2 presents family maltreatment by externalizing and internalizing variables. Analysis of variance (ANOVA) was conducted to examine the differences in externalizing and internalizing problems among the family maltreatment exposure groups. Externalizing problems significantly differed among the family maltreatment exposure groups $(\mathrm{F}=5.12, \mathrm{p}=.002)$. Those who experienced dual harm had higher levels of physical violence and aggression than those exposed to child maltreatment and interparental violence. Based on post-hoc t-tests between the groups (not shown), the only statistically significant results were between dual harm and interparental violence $(\mathrm{p}=.042)$, and dual harm and no maltreatment $(\mathrm{p}=.020)$. Differences between child maltreatment and no maltreatment approached statistical significance $(\mathrm{p}=.056)$. Internalizing problems also differed by family maltreatment $(\mathrm{F}=3.02, \mathrm{p}=.030)$ (not shown); that is, those who experienced dual harm had more internalizing problems than those only exposed to child maltreatment or interparental violence.

Table 2. Mean and standard deviation for family maltreatment by externalizing and internalizing behavior

\begin{tabular}{lcccc}
\hline & No maltreatment & $\begin{array}{c}\text { Interparental } \\
\text { violence }\end{array}$ & $\begin{array}{c}\text { Child } \\
\text { maltreatment }\end{array}$ & Dual Harm \\
\hline & $M(S D)$ & $M(S D)$ & $M(S D)$ & $M(S D)$ \\
Externalizing behavior & $1.09(2.02)$ & $2.92(4.82)$ & $5.58(5.75)$ & $6.09(5.54)$ \\
Internalizing behavior & $6.18(6.43)$ & $9.26(8.32)$ & $12.12(8.64)$ & $12.57(8.36)$ \\
\hline
\end{tabular}

\subsection{Examination of the Role of Social Support}

Two sets of hierarchical regression analyses were conducted to test effects of family maltreatment on college students' externalizing and internalizing problems and examine the role that social support from parents and close friends played in coping after family maltreatment. First, using the model by Aiken and West (1991), moderation analyses were conducted to see whether social support would interact with each predictor (i.e., the family maltreatment exposure group) to predict externalizing and internalizing problems. Moderating effects of social support were not significant for parents and friends (analysis not shown). Next, the mediating effects of social support were examined by following Baron and Kenny's (1986) method. Multiple regression analyses were conducted to test the relation between each predictor and mental health and behavioral problems with social support as a mediator. Mediating effects were not significant (analysis not shown). Effects of social support from parents and peers on externalizing and internalizing problems were neither moderating nor mediating, but direct (Research Question 1). 
Table 3 presents hierarchical regression analyses where externalizing behavior is the dependent variable. Model 1 included dummy variables for country (Hon Kong, South Korea, and Japan) and gender (man =1). These did not significantly predict externalizing behavior, $\mathrm{R}^{2}=.01, \mathrm{~F}(4,488)=1.04, \mathrm{p}>.05$.

Table 3. Summary of hierarchical regression analysis for predicting externalizing behaviors

\begin{tabular}{|c|c|c|c|c|c|c|c|c|c|}
\hline & \multicolumn{3}{|c|}{ Model 1} & \multicolumn{3}{|c|}{ Model 2} & \multicolumn{3}{|c|}{ Model 3} \\
\hline & $B(S E)$ & $\beta$ & $\mathrm{t}$ & $B(S E)$ & ß & $\mathrm{t}$ & $B(S E)$ & $\beta$ & $\mathrm{T}$ \\
\hline Hong Kong & $.18(.69)$ & .02 & .26 & $.25(.69)$ & .02 & .36 & $.01(.68)$ & .001 & .02 \\
\hline South Korea & $.21(.79)$ & .02 & .27 & $.44(.79)$ & .03 & .56 & $.74(.79)$ & .05 & .93 \\
\hline Japan & $-.42(.71)$ & -.03 & -.59 & $.21(.72)$ & .02 & .29 & $.17(.72)$ & .01 & .24 \\
\hline Men & $-.93(.57)$ & -.08 & -1.63 & $-1.02(.57)$ & $-.08+$ & -1.79 & $-.73(.57)$ & -.06 & -1.27 \\
\hline IV & & & & $1.17(2.17)$ & .04 & .54 & $1.15(2.15)$ & .04 & .54 \\
\hline $\mathrm{CM}$ & & & & $4.31(1.91)$ & $.36^{*}$ & 2.26 & $3.71(1.89)$ & $.31+$ & 1.96 \\
\hline Dual Harm & & & & $4.97(1.88)$ & $.44 * *$ & 2.65 & $4.40(1.86)$ & $.39 *$ & 2.37 \\
\hline SS Parents & & & & & & & $-.10(.04)$ & $-.14 * *$ & -2.73 \\
\hline SS Friends & & & & & & & $-.03(.04)$ & -.04 & -.86 \\
\hline $\mathrm{R}^{2}$ & & .01 & & & .04 & & & .07 & \\
\hline$\Delta \mathrm{R}^{2}$ & & .01 & & & .03 & & & .03 & \\
\hline
\end{tabular}

(IV=Interparental Violence; $\mathrm{CM}=$ Child maltreatment)

Note. The United States is the omitted category. $1=$ men and $0=$ women.

$+\mathrm{p}<.10 ; * \mathrm{p}<.05 ; * * \mathrm{p}<.01$

Model 2 added interparental violence, child maltreatment, and dual harm to Model 1 . This model significantly predicted externalizing behavior, $\mathrm{R}^{2}=.04, \mathrm{~F}(7,485)=2.87, \mathrm{p}<.01$. When compared to those not exposed, dual harm was the stronger predictor of externalizing behavior $(\beta=.44, \mathrm{p}=.008)$, followed by child maltreatment $(\beta=.36, \mathrm{p}=.024)$. Interparental violence was not statistically significant $(\beta=.04, \mathrm{p}=.59)$. There were no differences in externalizing behavior problems across countries or between men and women.

Model 3 added social support from parents and close friends to Model 2. This model significantly predicted externalizing behavior, $\mathrm{R}^{2}=.07, \mathrm{~F}(9,483)=3.72, \mathrm{p}<.001$. Dual harm was statistically significant, controlling for child maltreatment, interparental violence, parental and peer support, country of origin, and gender $(\beta=.39$, $\mathrm{p}=.018)$. Social support from parents was also statistically significant $(\beta=-.14, \mathrm{p}=.007)$. Child maltreatment was almost significant $(\beta=.31, \mathrm{p}=.051)$, while social support from close friends was an insignificant predictor $(\beta=-.04$, $\mathrm{p}=.39$ ).

Our findings indicate that those who experienced family violence reported higher levels of externalizing problems than those not exposed to any violence (Hypothesis 1). In addition, those who experienced dual harm were more likely to have externalizing problems than those exposed to any single form of violence (Hypothesis 2). Moreover, those who received higher levels of parental support experienced lower levels of external behavior problems (Research Question 2). Asian and Asian American college students benefitted from support from parents in reducing externalizing behavior problems, although effects of dual harm remained significant. Unlike parental support, peer supports did not contribute to reducing externalizing behavior. There were no significant differences in externalizing behavior among country of origin (Research Question 3).

The most important predictor of externalizing behavior problems was parental support, which uniquely explained $1.5 \%\left(\mathrm{sr}^{2}\right)$ of the variation in externalizing problems. Together, the nine independent variables accounted for $6.5 \%$ of the variance in externalizing problems. The proportion of the variance in externalizing behavior explained by the set of independent variables in Model $3\left(\mathrm{R}^{2}=6.5 \%\right)$ increased by $2.5 \%$ (Model 2), and by $5.7 \%$ (Model 1 ).

Table 4 shows the second set of hierarchical regression analyses with internalizing problems as the dependent variable. Model 1 included three sets of dummy variables for country of origin (Hong Kong, South Korea, and 
Japan) and gender $(\operatorname{man}=1)$. Model 1 did not significantly predict internalizing behavior, $\mathrm{R}^{2}=.01, \mathrm{~F}(4$, 487)=1.09, $\mathrm{p}>.05$.

Table 4. Summary of hierarchical regression analysis for predicting internalizing behaviors

\begin{tabular}{lccccccccc}
\hline & \multicolumn{3}{c}{ Model 1 } & \multicolumn{3}{c}{ Model 2 } & \multicolumn{3}{c}{ Model 3 } \\
\cline { 2 - 10 } Hong Kong & $B(S E)$ & $\beta$ & $\mathrm{T}$ & $B(S E)$ & $\beta$ & $\mathrm{t}$ & $B$ & $\beta$ & $\mathrm{t}$ \\
\cline { 2 - 10 } South Korea & $-.96(1.06)$ & -.05 & -.90 & $-.86(1.06)$ & -.05 & -.82 & $-1.38(1.03)$ & -.08 & -1.34 \\
Japan & $.89(1.21)$ & .04 & .73 & $1.21(1.21)$ & .06 & .99 & $1.61(1.19)$ & .08 & 1.36 \\
Men & $-.19(1.08)$ & -.01 & -.17 & $.54(1.10)$ & .03 & .49 & $.03(1.08)$ & .002 & .03 \\
IV & $.84(.87)$ & .05 & .97 & $.72(.87)$ & .04 & .83 & $1.71(.86)$ & $.09^{*}$ & 2.00 \\
$\mathrm{CM}$ & & & & $.90(3.35)$ & .02 & .27 & $.56(3.24)$ & .01 & .17 \\
Dual Harm & & & & $4.71(2.93)$ & .26 & 1.61 & $3.03(2.84)$ & .17 & 1.07 \\
SS Parents & & & & $5.64(2.88)$ & $.32+$ & 1.96 & $4.15(2.79)$ & .24 & 1.49 \\
SS Friends & & & & & & & $-.157(.06)$ & $-.15^{* *}$ & -2.88 \\
$\mathrm{R}^{2}$ & & & & & & & $-.201(.06)$ & $-.18^{* * *}$ & -3.53 \\
$\Delta \mathrm{R}^{2}$ & & .01 & & & .03 & & & .10 & .07 \\
\hline
\end{tabular}

(IV=Interparental Violence; CM=Child maltreatment)

Note. The United States is the omitted category. $1=$ men and $0=$ women.

$\mathrm{p}<.10+; \mathrm{p}<.05^{*} ; \mathrm{p}<.01 * * ; \mathrm{p}<.001 * * *$

Model 2 added interparental violence, child maltreatment, and dual harm to Model $1, \mathrm{R}^{2}=.03, \mathrm{~F}(7,484)=2.00$, p $>05$, which almost significantly predicted internalizing problems. Dual harm was almost statistically significant $(\beta=.32, \mathrm{p}=.051)$, but no other variables were significant. That is, students who had dual harm were slightly more likely to experience internalizing problems than those not exposed to any maltreatment.

Model 3 added social support from parents and close friends to Model 2, which significantly predicted internalizing problems, $\mathrm{R}^{2}=.10, \mathrm{~F}(9,482)=5.85, \mathrm{p}<.001$. By adding social support variables, the effects of dual harm on internalizing problems disappeared (Hypotheses 1 and 2.). Both social support from close friends $(\beta=-.18, p<.001)$ and social support from parents $(\beta=-.15, p=.004)$ contributed to the reduction of internalizing problems, controlling for family maltreatment, country of origin, and gender (Research Question 2). Asian and Asian American college students benefitted from parental and peer support in internalizing problems. However, there were no cross-cultural differences in externalizing and internalizing problems (Research Question 3).

The most important predictor of internalizing mental health concerns was social support from close friends, which uniquely explained $2.3 \%\left(\mathrm{sr}^{2}\right)$ of the variation in internalizing problems. Together, the nine independent variables accounted for $9.9 \%$ of the variance in internalizing problems. The proportion of variance in internalizing problems explained by a set of independent variables in Model $3\left(\mathrm{R}^{2}=9.9 \%\right)$ increased by $7.1 \%$ (Model 2), and by $9.0 \%$ (Model 1).

\subsection{Gender Differences in Internalizing Problems}

As demonstrated in Model 3 for Table 4, when the social support variable was added to Model 2 in the hierarchical regression model, gender was statistically significant for internalizing problems $(\beta=.09, p=.05)$. That is, men exhibited higher levels of internalizing mental health concerns than women. However, this conflicts with past findings that men cope with stress through externalizing behavior (e.g., anger and aggression) and that women internalize their behavior (e.g., feelings of guilt, depression, and anxiety; Stiles, Liu, \& Kaplan, 2000; Eschenbeck, Kohlman, \& Lohaus, 2008; Maschi, Morgen, Bradley, \& Hatcher, 2008). Likewise, Korean American adolescent men demonstrated more rule-breaking behavior (Shrake \& Rhee, 2004; Kim, Chen, Kools, \& Weiss, 2016).

To explore this further, we conducted simple regression analyses between social support and internalizing problems by gender. We found that Asian and Asian American men received lower levels of parental support 
( $\beta=-.14, p=.001)$ and peer support $(\beta=-.19, p=.001)$ (results not shown) than their women counterparts. Social support is expected to help college students cope with physical and mental stress and stressful life situations (Talwar \& Mohd Fadzil, 2013). Less parental and peer support experienced by Asian and Asian American men increased mental stress and minimized the role of a protective factor for psychological distress. Iwamoto, Liao, and Liu (2010) explained higher levels of internalizing problems among men. Specifically, Asian American men who endorse dominance for masculine scripts (unlike winning norms, a protective factor) reported more depression symptoms. Adhering to dominance masculine norms may create distress in relationships.

\section{Discussion}

As expected, Asian and Asian American college students exposed to dual harm, compared with those with no maltreatment, showed more externalizing behavior, but no difference in internalizing problems for these comparisons. Participants exposed to dual harm showed more externalizing behavior than those exposed to one type of maltreatment. However, dual harm and any single type of maltreatment had no differences in internalizing problems. This result was similar to Han et al.'s (2016) outcomes, which found that South Korean college students who were exposed to a single form of violence showed equally high levels of internalizing problems as those exposed to dual harm.

Initially, effects of dual harm on externalizing problems were stronger than for any other group. However, when social support was entered in the regression model, the effect of dual harm weakened, but remained significant. The effect of parental support was strong, but peer support was not significant, indicating that Asian and Asian American college students' behavioral problems were reduced mainly from the inclusion of parental support. Although past studies showed that adolescents spend less time with parents and rely on their peers for support (Feiring et al., 1998), and minimize help-seeking from family members (Meyers, 2006), Folger and Wright (2013) noted that among college students, social support from family helps reduce anger and hostility.

Additional findings here were that both parental and peer support helped reduce internalizing problems, and that peer support was a stronger effect than that of parental support. The effect of dual harm disappeared when the social support variable entered in the regression model. Although Asian cultural values discourage maltreated individuals from seeking support from parents and friends, our findings imply that our college participants received and benefited from their support.

Our findings concur with Folger and Wright's (2003) work on college aged men and women and Lee and Hadeed's (2009) examination of South Korean immigrants, which showed those who received social support from families and friends coped better with life stress and distress symptoms. Holahan et al. (1995) found a negative relationship between parental support and psychological problems, while Bal et al. (2003) suggested that the comfort of peer support was not enough to avoid internalizing problems.

There were no cross-cultural differences in externalizing and internalizing problems, which indicated levels of both problems were similar among four countries. Although within-group differences are known to exist among Asian countries, researchers like Anderson and Mayes (2010), Kim, Atkinson, and Yang (1999), and Okumura et al. (2016) maintained there are a number of cultural similarities among Asian ethnic groups (see Literature Review). Further, there are no differences in levels of anxiety and depression between Chinese and Japanese Americans (Okumura et al., 2016). However, due to the limited number of studies on similarities in the effects of family violence and maltreatment, we found it difficult to make cross-cultural comparisons for externalizing and internalizing problems.

\section{Study Limitations}

We carefully collected data from four countries; however, issues with cross-cultural data collection might have affected the findings. For example, there were differences in timing and procedures of data collection. In Asian countries, collaborators collected data during class time using hard copies of the survey, while in the United States, Asian American students completed the survey online. Also, although key concepts used in the survey instrument were carefully examined and chosen, the assumption of conceptual equivalence-defined as a construct having the same meaning across cross-cultural groups-may be problematic when comparisons are made across less similar contexts (i.e., Asian countries and the United States; Harachi. Choi, Abbott, Catalano, \& Bliesner, 2006).

Moreover, selection of the universities and countries included in the study was somewhat biased, since the universities were not chosen randomly and the selected countries do not represent all Asian countries. The participants were drawn using a convenience sample and may not represent all college students in each country. 
Further, Asian American student immigration status, ethnic backgrounds, and length of stay in the United States were not collected, impeding comparisons.

Our hierarchical regression analyses accounted for small amounts of variance in externalizing problems and internalizing problems, indicating that the set of independent variables included in our study did not have stronger explanatory power. Perhaps other variables are better predictors of externalizing and internalizing problems, such as childhood temperament, family environment (Leve, Kim, \& Pears, 2005), and parenting styles (Akhter, Hanif, Tariq, \& Atta, 2011). Chengliang (2011) showed that Chinese parents are more authoritarian than American parents based on Confucian ideas, although he did not suggest that Chinese parenting styles increased internalizing and externalizing problems.

As Chan (2015) and Gewirtz and Edleson (2004) maintained, a cross-sectional study does not provide causality, it only shows a correlation among variables. As our study was cross-sectional and participants were not asked which problems they encountered first (exposure to interparental violence or child maltreatment), we were not able to explore the temporal order. Further, we could not assess exactly how college students have been affected by family violence at each developmental stage and how protective factors served to help participants cope with externalizing and internalizing problems despite exposure to risks. As per social learning theory, it is possible that college students have learned cultural traditions, beliefs, and norms-including violent ideas-from their families and peers in childhood, which remained until adulthood. Therefore, longitudinal studies are required to understand the differences between exposed and non-exposed groups.

\section{Conclusion}

The present study provided new insights into family violence and maltreatment and its long-term effects on Asian and Asian American college students' externalizing and internalizing problems and the role of social support in dealing with stress events. Our findings indicated that exposure to dual harm was more detrimental than effects of a single type of maltreatment to college students' behavioral functioning.

Further, the role of social support was directly related to externalizing and internalizing behavior. Both support from parents and close friends are found to be key elements in reducing internalizing mental health problems in response to family violence, while support from parents only was found to reduce externalizing behavior. Because of cultural constraints, Asian and Asian American families and friends are viewed as strain rather than support. In our study, social support was the source of support. Indeed, social support appeared to have lasted until our participants became college students.

The Asian American and Asian immigrant populations in the United States have grown over the past few decades ("U. S. Census Bureau," n.d.; Zong \& Batalova, 2016). Despite this growth, this area of research among Asian Americans is still limited, and further investigation of family maltreatment associated with various circumstances, such as family immigration backgrounds, is needed.

\section{Acknowledgement}

The authors would like to acknowledge Dr. Meekuyng Han in the School of Social Work at San José State University and international collaborators (Professors Sing Lau at Hong Kong Baptist University, Hong Kong, Young Choi at Chung-Ang University, South Korea, Soyon Jung at Seoul Women's University, South Korea, Takashi Arai at Otemon Gakuin University, Japan, Etsuko Maruyama at Tokiwa University, Japan, and Harumi Miyagi at Okinawa University, Japan) for their valuable support for this project.

\section{References}

Achenbach, T. M. (1991). Manual for the Child Behavior Checklist/4-18 and 1991 profile. Burlington, VT: University of Vermont Department of Psychiatry.

Aiken, L. S., \& West, S. G. (1991). Multiple regression: Testing and interpreting interaction. Thousand Oaks, CA: Sage.

Akers, R. (1998). Social learning and social structure: A general theory of crime and deviance. Boston, MA: Northeastern University Press.

Akhter, N., Hanif, R., Tariq, N., \& Atta, M. (2011). Parenting styles as predictors of externalizing and internalizing behavior problems among children. Pakistan Journal of Psychological Research, 26(1), 23-41.

Anderson, E. R., \& Mayes, L. C. (2010). Race/ethnicity and internalizing disorders in youth: A review. Clinical Psychology Review, 30(3), 338-348. 
Appel, A. E., \& Holden, G. W. (1998). The co-occurrence of spouse and physical child abuse: A review and appraisal. Journal of Family Psychology, 12(4), 578-599.

Bal, S., Crombez, G., Van Oost, P., \& Debourdeaudhuij, I. (2003). The role of social support in well-being and coping with self-reported stressful events in adolescents. Child Abuse \& Neglect, 27(12), 1377-1395.

Baron, R. M., \& Kenny, D. A. (1986). The moderator-mediator variable distinction in social psychological research: Conceptual, strategic, and statistical considerations. Journal of Personality and Social Psychology, 51, 1173-1182.

Baumeister, R. F., \& Leary, M. R. (1995). The need to belong: desire for interpersonal attachments as a fundamental human motivation. Psychological bulletin, 117(3), 497.

Bokhorst, C. L., Summer, S. R., \& Westenberg, P. M. (2010). Social Support from parents, friends, classmates, and teachers in children and adolescents aged 9 to 18 years: Who is perceived as most supportive?. Social Development, 19(2), 417-426.

Bouhours, B., \& Broadhoust, R. (2015). Violence Against Women in Hong Kong: Results of the International Violence Against Women Survey. Violence Against Women, 21(11), 1311-1329.

Carr, J. L., \& Vandeusen, K. M. (2002). The relationship between family origin violence and dating violence in college men. Journal of Interpersonal Violence, 17(6), 630-646.

Chan, K. L. (2011). Children exposed to child maltreatment and intimate partner violence: A study of co-occurrence among Hong Kong Chinese families. Child Abuse \& Neglect, 35(7), 532-542.

Chan, K. L. (2015). Association between intimate partner violence and child maltreatment in a representative student sample in Hong Kong. Violence Against Women, 21(4), 500-515.

Chen, X., Liu, M., Rubin, K. H., Li, D., Li, Z., Cen, G., \& Li, B. (2002). Parental reports of externalizing and internalizing behaviors in Chinese children: Relevancy to social, emotional and school adjustment. Journal of Psychology in Chinese Societies, 3, 233-260.

Chengliang, Z. (2011). Praise or criticism? ChinaDaily.com, 18. Retrieved from http://www.chinadaily.com.cn/cndy/2011-08/30/content_13215642.htm

Cho, H. (2012). Intimate Partner Violence among Asian Americans: Risk factor differences across ethnic subgroups. Journal of Family Violence, 27, 215-224

Cobb, S. (1976). Presidential Address - 1976. Social support as a moderator of life stress. Psychosomatic Medicine, 38(5), 300-314.

Davidson, L. M., \& Damaray, M. K. (2007). Social Support as a Moderating Between Victimization and Internalizing-Externalizing Distress From Bullying. School Psychology Review, 36(3), 383-405.

Eriksson, L., \& Mazerolle, P. (2015). A cycle of violence? Examining family-of-origin violence, attitudes, and intimate partner violence perpetration. Journal of Interpersonal Violence, 30(6), 945-964.

Eschenbeck, H., Kohlman, C. W., \& Lohaus, A. (2007). Gender differences in coping strategies in children and adolescents. Journal of Individual Differences, 28(1), 28-26. https://doi.org/10.1027/1614-0001.28.1.18

Ezzell, C. E., Swenson, C. C., \& Brondino, M. J. (2000). The relationship of social support to physically abused children's adjustment. Child Abuse \& Neglect, 24(5), 641-651.

Feiring, C., Taska, L. S., \& Lewis, M. (1998). Social support and children's and adolescents' adaptation to sexual abuse. Journal of Interpersonal Violence, 13(2), 240-260.

Ferguson, K. M. (2009) Exploring family environment characteristics and multiple abuse experiences among homeless youth. Journal of Interpersonal Violence, 24(11), 1875-1891.

Finkelhor, D., Turner, H., Ormrod, R., Hamby, S., \& Kracke, K. (2009). Children's exposure to violence: A comprehensive national survey. Juvenile Justice Bulletin. Washington, D.C.: United States Department of Justice.

Folger, S. F., \& Wright, M. O’D. (2013). Altering risk following child maltreatment: Family and friend support as protective factors. Journal of Family Violence, 28, 325-337.

Gewirtz, A., \& Edleson, J. L. (2004). Young children's exposure to adult domestic violence: Toward a developmental risk and resilience framework for research and intervention. In S. Schechter (Ed.), 
Developmental risk and resilience framework for research and intervention (pp. 1-27). Iowa City, IA: The University of Iowa. Retrieved from http://www.uiowa.edu/ socialwk/publications.html

Gilbert, R., Widom, C. S., Browne, K., Fergusson, D., Webb, E., \& Janson, S. (2009). Burden and consequences of child maltreatment in high-income countries. The Lancet, 373, 68-81.

Gover, A. R., Jennings, W. G., Tomsich, E. A., Park, M., \& Rennison, C. M. (2011). The influence of childhood maltreatment and self-control on dating violence: A comparison of college students in the United States and South Korea. Violence and Victims, 26(3), 296-318.

Haj-Yahia, M. M., \& de Zoysa, P. (2008). Rates and psychological effects of exposure to family violence among Sri Lankan university students. Child Abuse \& Neglect, 32, 994-1002.

Hamburger, M. E., Leeb, R. T., \& Swahn, M. H. (2008). Childhood maltreatment and early alcohol use among high-risk adolescents. Journal of Studies on Alcohol \& Drugs, 69(2), 291-295.

Han, M., Choi, Y., \& Jung, S. (2016). Exploring the relationship between exposure to interparental violence and child physical abuse in childhood and the impacts on mental health problems in later young adulthood among South Korean college students. International Social Work, 59(6), 821-835. https://doi.org/10.1177/0020872814562481

Harachi. T. W., Choi, Y., Abbott, R. D., Catalano, R. F., \& Bliesner, S. L. (2006). Examining equivalence of concepts and measures in diverse samples. Prevention Science, 7(4), 359-368. https://doi.org/10.1007/s11121-006-0039-0

Heise, L., \& Garcia-Moreno, C. (2002). Violence by intimate partners. In E. G. Krung, L. L. Dahlberg, J. A. Mercy, A. B. Zwi, \& R. Lozano (Eds.), World report on violence and health (pp. 87-121). Geneva, Switzerland: World Health Organization (WHO).

Herrenkohl, T. I., Sousa, C., Tajima, E. A., Herrenkohl, R. C., \& Mylan, C. A. (2008). Intersection of child abuse and children's exposure to domestic violence. Trauma, Violence \& Abuse, 9(2), 84-99.

Herrenkohl, T., Jung, H., Klika, J. B., Mason, W. A., Brown, E. C., Leeh, R. T., Herrenkohl, R. C. (2016). Mediating and Moderating Effects of Social Support in the Study of Child Abuse and Adult Physical and Mental Health. American Journal of Orthopsychiatry, 86(5), 573-583. https://doi.org/10.103//ort0000136.

Hicks, M. H. (2006). The prevalence and characteristics of intimate partner violence in a community study of Chinese American women. Journal of Interpersonal Violence, 10, 1249-1269.

Higgins, D. J., \& McCabe, M. P. (2001). Multiple forms of child abuse and neglect: Adult retrospective reports. Aggression and Violent Behavior, 6, 547-578.

Holahan, C. J., Valentiner, D. P., \& Moos, R. H. (1995). Parental support, coping strategies, and psychological adjustment: An integrative model with late adolescents. Journal of Youth and Adolescence, 24, 633-648.

Isaac, L. (2015, July 29). The origins and practice of Trauma-Focused Cognitive Behavioral Therapy (TF-CBT). Social Justice Solutions. Retrieved from http://www.socialjusticesolutions.org/2015/07/29/origins-practice-trauma-focused-cognitive-behavioral-th erapy-tf-cbt/

Iwamoto, D. K., Liao, L., \& Liu, W. M. (2010). Masculine norms, avoidant coping, Asian values, and depression Among Asian American men. Psychology of Men \& Masculinity, 11(1), 15-24.

James, A., \& Mennen, F. (2018, January). The Mediating Effects of Social Support on Maltreatment and Internalizing Problems Among Adolescents. ePoster session presented at the Annual Conference of Society for Social Work and Research, Washington, D.C.

Keen, B., Pickering, D., Wieczorek, M., \& Blaszczynski, A. (2015). Problem gambling and family violence in the Asian context: a review. Asian Journal of Gambling Issues and Public Health, 5(3). https://doi.org/10.1186/s40405-015-0008-2

Kennedy, A. C., Bybee, D., Sullivan, C. M., \& Greeson, M. (2010). The Impact of family and community violence on children's depression trajectories: Examining the interactions of violence exposure, family social support, and gender. Journal of Family Psychology, 24(2), 197-207.

Kiesel, L. R., Piescher, K. N., \& Edleson, J. L. (2016). The relationship between child maltreatment, intimate partner violence exposure, and academic performance. Journal of Public Child Welfare, 10(4), 434-456. 
Kim, B. (2018, May 20). Hierarchical Linear Regression. University of Virginia. Library. Retrieved from https://data.library.virginia.edu/hierarchical-linear-regression/

Kim, B. S. K., Atkinson, D. R., \& Yang, P. H. (1999). The Asian Values Scale: Development, factor analysis, validation, and reliability. Journal of Counseling Psychology, 46, 342-352.

Kim, H. S., Sherman, D. K., \& Taylor, S. E. (2008). Culture and Social Support. American Psychologist, 63(6), 518-526. https://doi.org/10.1037/0003-066X

Kim, J., \& Sung, K. (2000). Conjugal violence in Korean American families: A residue of the cultural tradition. Journal of Family Violence, 15(4), 331-345.

Kim, M., Chen, J-L., Kools, S., \& Weiss, S. (2016). Mental health problems among Korean American adolescents. Psychology, 7, 1872-1882.

Kopp, R. (2010). Saving Face: A little discretion can go a long way in Japan. Chicago, Illinois: Japan Intercultural Consulting. Retrieved from http://www.japanintercultural.com/en/news/default.aspx?newsID=59

Lee, E. (2007). Domestic violence and risk factors among Korean immigrant women in the United States. Journal of Family Violence, 22(3), 141-149.

Lee, J. Y., Chung, I. J., Back, J. L., \& Batzolboo, B. (2014). The similarities and differences in predictor variables of youth's prosocial and antisocial behaviors: A meta-analysis. Journal of Korean Society of Child Welfare, 47, 125-155.

Lee, Y-S., \& Hadeed, L. (2009). Intimate partner violence among Asian immigrant communities: Health/mental health consequences, help-seeking behaviors, and service utilization. Trauma, Violence, \& Abuse, 10(2), 143-170.

Leve, L. D., Kim, H. K., \& Pears, K. C. (2005). Childhood temperament and family environment as predictors of internalizing and externalizing trajectories from age 5 to 17. Journal of Abnormal Child Psychology, 33(5), 505-520.

Luster, T., Small, S. A., \& Lower, R. (2002). The correlates of abuse and witnessing abuse among adolescents. Journal of Interpersonal Violence, 17(12), 1323-1340.

Malecki, C. K., \& Demaray, M. K. (2002). Measuring perceived social support: Development of the Child and Adolescent Social Support Scale (CASSS). Psychology in the Schools, 39(1), 1-18.

Maschi, T., Morgen, K., Bradley, C., \& Hatcher, S. S. (2008). Exploring Gender Differences on Internalizing and Externalizing Behavior Among Maltreated Youth: Implications for Social Work Action. Child Adolescent Social Work Journal, 25, 531-547. https://doi.org/10.1007/s10560-008-0139-8

Matsuda, T., Tsuda, A., Kim, E., \& Deng, K. (2014). Association between perceived social support and subjective well-being among Japanese, Chinese, and Korean college students. Psychology, 5, 491-499. https://doi.org/10.4236/psych.2014.56059

McCloskey, L. A., Figueredo, A. J., \& Koss, M. P. (1995). The effects of systemic family violence on children's mental health. Child Development, 66, 1239-1261. https://doi.org/10.1111/j.1467-8624.1995.tb00933.x

Meyers, L. (2006). Asian-American mental health. American Psychological Association, 37(2), 44. Retrieved from http://www.apa.org/monitor/feb06/health.aspx

Moylan, C. A., Herrenkohl, T. I., Sousa, C., Tajima, E. A., Herrenkohl, R. C., \& Russo, M. J. (2010). The effects of child abuse and exposure to domestic violence on adolescent internalizing and externalizing behavior problems. Journal of Family Violence, 25(1), 53-63.

Okumura, K. L., Ebesutani, C., Bloom, R., Higa-McMillan, C. K., Nakamura, B., \& Chorpita, B. F. (2016). Differences in internalizing symptoms across specific ethnic minority groups: An analysis across Chine American, Filipino American, Japanese American, Native Hawaiian, and White youth. Journal of Child and Family Studies, 25(11), 3353-3366.

Owen, A. E., Thompson, M. P., Mitchell, M. D., Kennebrew, S. Y., Paranjape, A., Reddick, T. L., ... Kaslow, N. J. (2008). Perceived social support as a mediator of the link between intimate partner conflict and child adjustment. Journal of Family Violence, 23, 221-230. https://doi.org/10.1007/s10896-007-9145-4 
Parker, J. G., \& Asher, S. R. (1993). Friendship and friendship quality in middle childhood: Links with peer group acceptance and feelings of loneliness and social dissatisfaction. Developmental Psychology, 29, 611-621.

Salzinger, S., Feldman, R. S., Ng-Mak, D. S., Mojica, E., Stockhammer, T., \& Rosario, M. (2002). Effects of partner violence and physical child abuse on child behavior: A study of abused and comparison children. Journal of Family Violence, 17(1), 23-52.

Sangalang, C. C., \& Gee, G. C. (2012). Depression and anxiety among Asian Americans: The effects of social support and strain. Social Work, 57(1), 49-60.

Schumm, J. A., Briggs-Phillips, M., \& Hobfoll, S. E. (2006). Cumulative interpersonal traumas and social support as risk and resiliency factors in predicting PTSD and depression among inner-city women. Journal of Traumatic Stress, 19, 825-836. https://doi.org/10.1002/jts.20159

Shen, A. C. T. (2009). Self-esteem of young adults experiencing interpersonal violence and child physical maltreatment: parental and peer relationships as mediators. Journal of Interpersonal Violence, 24(5), 770-794.

Shrake, E. K., \& Rhee, S. (2004). Ethnic identity as predictor of problem behaviors among Korean American adolescents. Adolescence, 39, 601-622. SONA online system (n.d.). Retrieved from http://www.sona-systems.com/about.aspx

Stiles, B. L., Liu, X., \& Kaplan, H. B. (2000). Relative deprivation and deviant adaptations: The mediating effects of negative self-feelings. Journal of Research in Crime and Delinquency, 37, 64-90. https://doi.org/10.1177/0022427800037001003

Stiles, M. (2002). Witnessing domestic violence: The effect on children. American Family Physician, 66, 2052-2058.

Straus, M. A., \& Smith, C. (1990). Family patterns and child abuse. In M. A. Straus \& R. J. Gelles (Eds.), Physical violence in American families: Risk factors and adaptations to violence in 8,145 families (pp. 507-525). New Brunswick, NJ: Transaction Publishing.

Straus, M.A., Hamby, S. L., Boney-McCoy, S., \& Sugarman, D. B. (1996). The revised Conflict Tactics Scales (CTS2): Development and preliminary psychometric data. Journal of Family Issues, 17(3), 283-316.

Straus, M. A., Hamby, S. L., Finkelhor, D., Moore, D. W., \& Runyan, D. (1998). Identification of child maltreatment with the Parent-Child Conflict Tactics Scales: development and psychometric data for a national sample of American parents. Child Abuse \& Neglect, 22(4), 249-270.

Talwar, P., \& Mohd Fadzil, A. R. (2013). Perceived social support among university students in Malaysia: A reliability study. ResearchGate. Retrieved from https://www.researchgate.net/publication/236160288

Taylor, S. E., Sherman, D. K., Kim, H. S., Jarcho, J., Takagi, K., \& Dunagan, M. S. (2004). Culture and Social Support: Who Seeks It and Why? Journal of Personality and Social Psychology, 87(3), 354-362. https://doi.org/10.1037/0022-3514.87.3.354

Thoits, P. A. (2011). Mechanisms linking social ties and support to physical and mental health. Journal of Health and Social Behavior, 52(2), 145-161.

Tremblay, C., Hébert, M., \& Piché, C. (1999). Coping strategies and social support as mediators of consequences in child sexual abuse victims. Child Abuse \& Neglect, 23(9), 929-945.

Tsokodayi, C. (2010). Violence against women cuts across income, class and culture lines - UN Report. Washington D.C.: United Nations. Retrieved from http://www.examiner.com

U.S. Department of Health and Human Services. (2018). Effects of violence against women. Washington, D.C.: Office on Women's Health. https://www.womenshealth.gov/relationships-and-safety/effects-violence-against-women

UNICEF. (2014). Hidden in Plain Sight: A statistical analysis of violence against children. New York, NY: UNICEF. http://files.unicef.org/publications/Hidden_in_Plain_sight_statistical_analysis_EN_3_Sept_2014.pdf

United States Census Bureau (n.d.). Quick facts United States population estimates 2018. Retrieved from https://www.census.gov/quickfacts/fact/table/US/PST045218 
Vernberg, E. M., La Greca, A. M., Silverman, W. K., \& Prinstein, M. J. (1996). Prediction of posttraumatic stress symptoms in children after Hurricane Andrew. Journal of Abnormal Psychology, 105, 237-248.

White, R., \& Ren, K. (2012). Externalizing behavior problems during adolescence: An ecological perspective. Journal of Child and Family Studies, 21, 158-171.

Wilson, H. W., Stover, C. S., \& Berkowitz, S. J. (2009). Research review: The relationship between childhood violence exposure and juvenile antisocial behavior: A meta-analytic review. Journal of Child Psychology and Psychiatry, 50(7), 769-779.

Wolfe, D. A., Crooks, C. V., Lee, V., McIntyre-Smith, A., \& Jaffe, P. G. (2003). The effects of children's exposure to domestic violence: A meta-analysis and critique. Journal of Clinical Family Psychological Review, 6(3), 171-197.

Yick, A. G. (2000). Predictors of physical spousal/intimate violence in Chinese American families. Journal of Family Violence, 15(3), 249-267.

Yoshihama, M. (1999). Domestic violence against women of Japanese descent in Los Angeles: two methods of estimating prevalence. Violence Against Women, 5, 869-897.

Yoshihama, M. (2002). Battered women's coping strategies and psychological distress: differences by immigration status. American Journal of Community Psychology, 30(3), 429-452. https://doi.org/10.1023/A:1015393204820

Yoshihama, M., \& Dabby, C. (2009, September). Facts \& stats: Domestic violence in Asian, Native Hawaiian and Pacific Islander homes. San Francisco, CA: Asian Pacific Islander Institute on Domestic Violence.

Yoshihama, M., \& Horrocks, J. (2003). The Relationship Between Intimate Partner Violence and PTSD? An Application of Cox Regression with Time-Varying Covariates. Journal of Traumatic Stress, 16, 371-380. https://doi.org/10.1023/A:1024418119254

Zong, J., \& Batalova, J. (2016). Asian immigrants in the United States. Washington, D.C. Migration Policy Institute. Retrieved from https://www.migrationpolicy.org/article/asian-immigrants-united-states

\section{Note}

Note 1. Overseas colleagues were solicited directly and indirectly through the first author's network of researchers.

\section{Copyrights}

Copyright for this article is retained by the author(s), with first publication rights granted to the journal.

This is an open-access article distributed under the terms and conditions of the Creative Commons Attribution license (http://creativecommons.org/licenses/by/4.0/). 